\title{
Experimental Study on Jet Formation and Penetration Characteristics of Square cross-section Shaped Charge
}

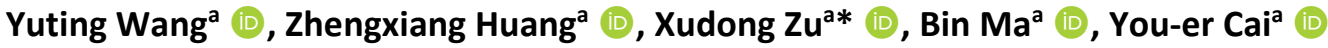 \\ a School of Mechanical Engineering, Nanjing University of Science and Technology, Nanjing 210094, China. E-mails: \\ wangyuting@njust.edu.cn; huangyu@njust.edu.cn; zuxudong9902@njust.edu.cn; mabin@njust.edu.cn; caiyouer@njust.edu.cn \\ * Corresponding author.
}

https://doi.org/10.1590/1679-78256514

\begin{abstract}
The jet morphology of square and circular cross-section shaped charge with same inscribed circle diameter were observed by conducting an X-ray experiment. Depth-of-penetration tests at standoffs 80 and $160 \mathrm{~mm}$ were also carried out. The jet formation was studied by using AUTODYN software. The results showed that the jet of square cross-section shaped charge consists of a condensed part and a non-condensed part. The condensed part has similar characteristics to the corresponding part of circular cross-section shaped charge. And the non-condensed part is distributed at the jet tail. The non-condensed part consists of a main fluid moving along the axis and four off-axis fluids with off-axis velocity along the symmetry plane. The noncondensing of jet reduces the penetration depth of square cross-section shaped charge, the decreasing rate increased from $2.67 \%$ to $17.3 \%$ when the standoff is increased from $80 \mathrm{~mm}$ to $160 \mathrm{~mm}$. When the inscribed circle diameter of the charge cross-section increases to $67.2 \mathrm{~mm}$ (1.2 times of $56 \mathrm{~mm}$ ) and the liner structure remains the same, the square cross-section shaped charge will form a cohesive jet.
\end{abstract}

\section{Keywords}

square cross-section; shaped charge; jet formation; penetration

\section{Graphical Abstract}

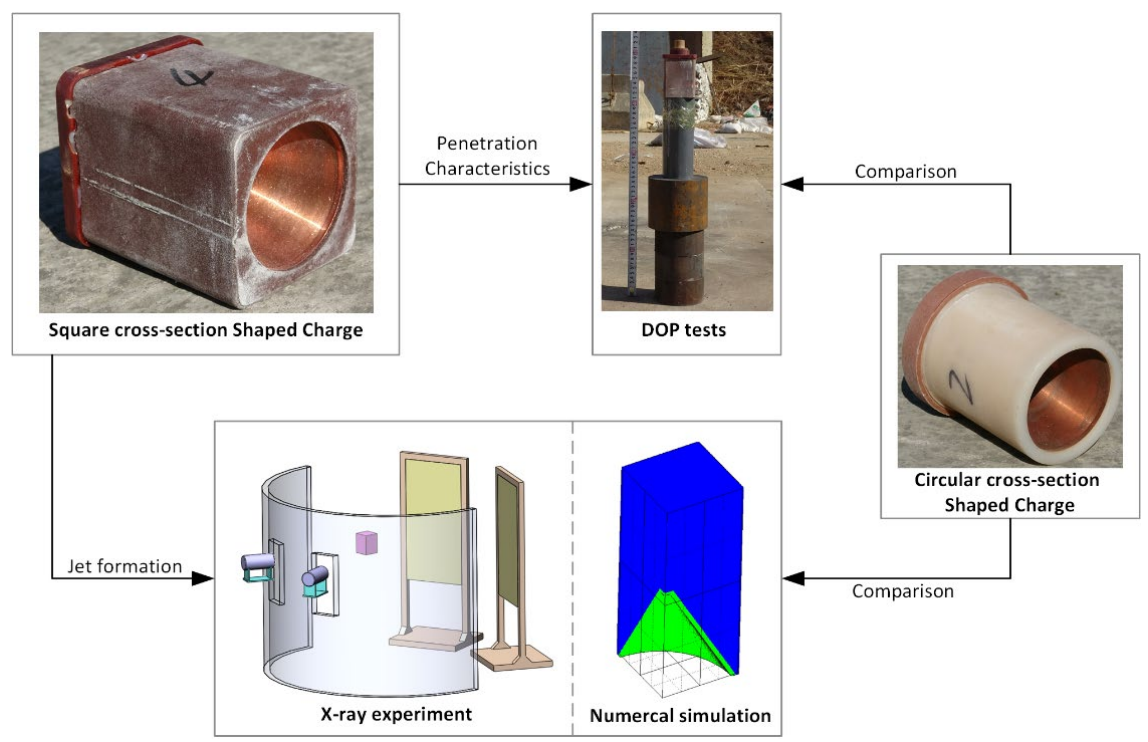




\section{INTRUDUCTION}

Square cross-section shapes are widely used in aerial weapon platforms [1-2]. When these platforms adopt a shaped charge warhead, they could also effectively destroy armored targets and fortifications even if they reach the target at a low residual velocity. At present, the shaped charge mainly adopts a rotationally symmetric structure [3]. However, square cross-section aerial weapon platforms adopt shaped charge with rotationally symmetric structure, which could result in a low explosive charge fill ratio and limit the destruction caused by warheads. Thus, the use of square crosssection shaped charge in aerial weapon platforms with a square cross-section body could become a future trend.

At present, some studies have been conducted on square cross-section shaped charges. Cullis [4] studied the jet formation of a square cross-section shaped charge with a square-based pyramidal liner. The results showed that as the observation position moves from the slug to the jet, the characteristic of the star cross-section rapidly thins down to a nearly circular section. Wang [5] studied the jet formation and penetration characteristics of the square cross-section shaped charge with rotationally symmetric structure liner by using ANSYS-LSDYNA software. The results showed that the cross-section shape of the front segment of the jet is close circular, while that of the back segment of the jet is four-angle star, and the shaped charge in this work had a high penetration ability.

Existing studies on square cross-section shaped charge were mainly based on numerical calculation. Limited experimental works were carried out to investigate the jet formation and penetration characteristics of square crosssection shaped charge. Therefore, the present paper aimed to fill this gap. Here, the jet morphology of square crosssection shaped charge with inscribed circle diameters of $56 \mathrm{~mm}$ was observed by conducting an X-ray experiment, depthof-penetration (DOP) tests at standoffs 80 and $160 \mathrm{~mm}$ were also carried out. The experimental results are compared with the circular cross-section shaped charge with same inscribed circle diameter. In addition, to clarify the jet formation of shaped charges, related numerical simulation studies were carried out on the basis of smoothed particle hydrodynamic (SPH) method. The research conclusion has a reference value for the application of square cross-section shaped charge warhead in the square cross-section aerial weapon platform.

\section{EXPERIMENT}

\subsection{Structure of shaped charge}

Figure 1 shows the square and circular cross-section shaped charges. The liner adopts the same structure: a single cone structure with a cone angle of $60^{\circ}$, the liner diameter is $54.2 \mathrm{~mm}$, the height is $42 \mathrm{~mm}$, and the thickness is $1 \mathrm{~mm}$. The inscribed circle diameter of the charge cross-section of the square cross-section shaped charge is $56 \mathrm{~mm}$, and the height of the charge is $73.3 \mathrm{~mm}$. The diameter of the circular cross-section shaped charge is $56 \mathrm{~mm}$, and the height of the charge is also $73.3 \mathrm{~mm}$.

The materials of the explosive and liner are Composition B and OFHC Cu, respectively. A $3 \mathrm{~mm}$-thick nylon shell was used to reduce the difficulty of charge casting and ensure the characteristic size of the charge. Once the detonation wave reaches the interface between the charge and the shell, the surface rarefaction wave enters into the explosive products, just like the shaped charge without a shell. The state of the detonation products at different times in the shaped charge with a thin nylon shell is the same as that in the shaped charge without a shell. Therefore, the shaped charge in this work could be regarded as shaped charges without a shell.

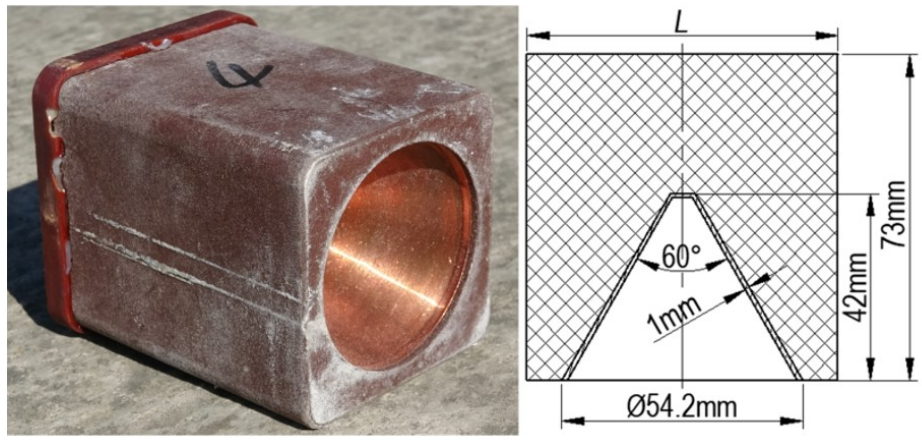

(a) square cross-section shaped charge
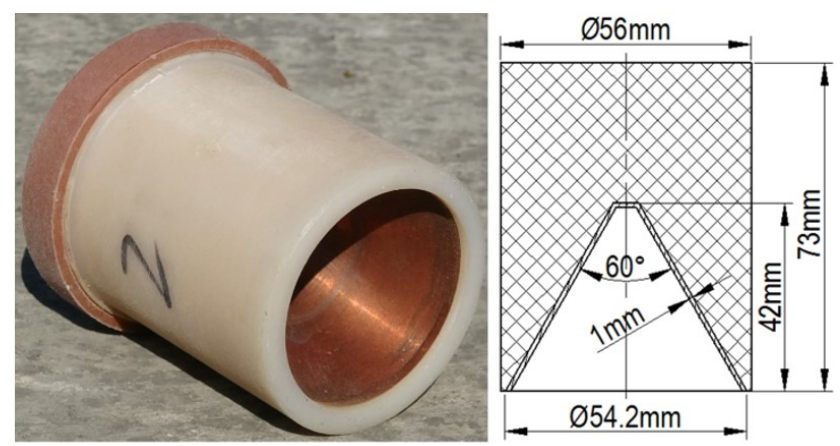

(b) circular cross-section shaped charge

Figure 1 Physical photographs of shaped charges. 


\subsection{X-ray experimental setup}

The setup of the X-ray experiment is shown in Figure 2. The jet motion was recorded using a pulse X-ray photography system, which is combined by two pulse X-ray tubes. X-ray tubes A and B were placed at an angle of $45^{\circ}$. The shaped charge was placed in the intersection area of the two $X$-ray tubes, and the shaped charge was parallel to the ground. Two pulsed X-ray tubes worked at different times to obtain X-ray photos at different times.

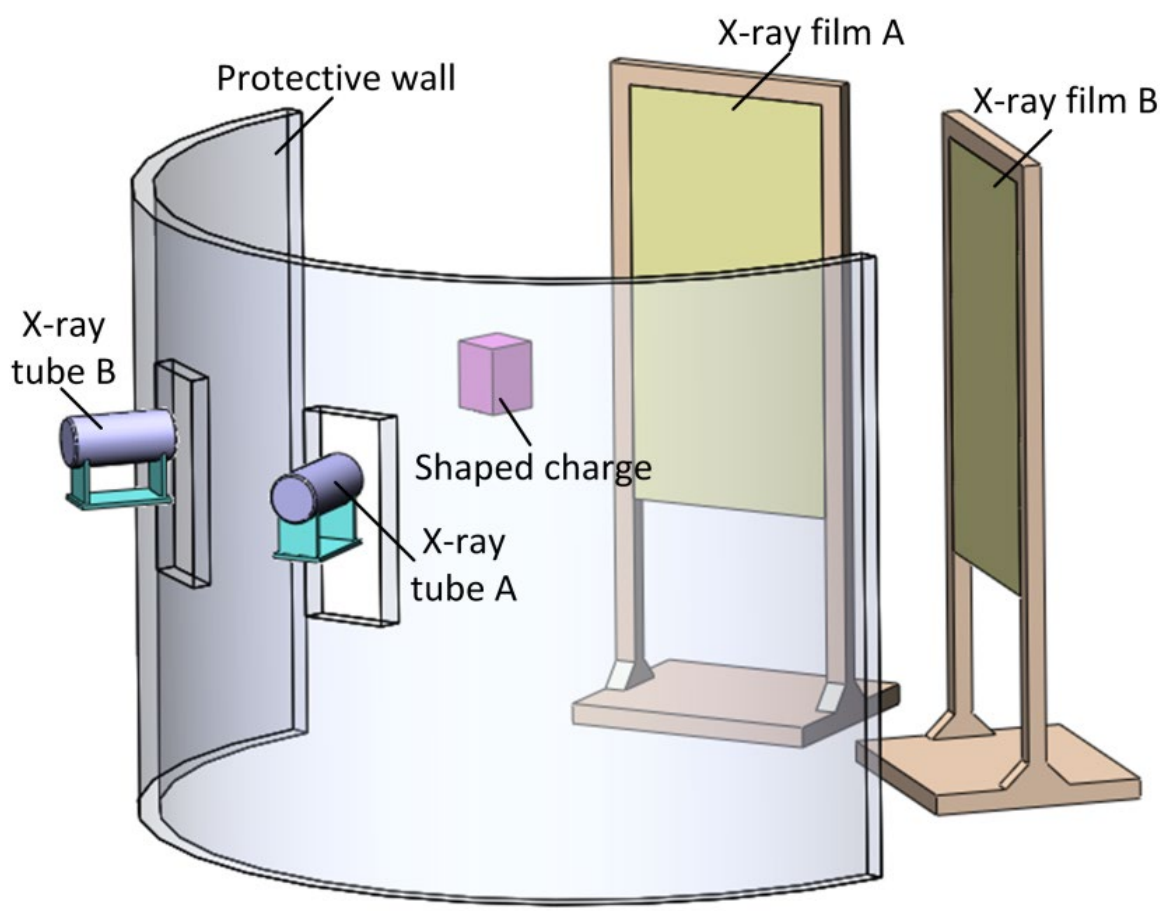

Figure 2 X-ray experimental configuration and setup.

The relative position of the configurations in the X-ray experiment is shown in Figure 3 . The photo taken by the Xray tube $A$ reflects the diagonal view of the square cross-section shaped charge, and that taken by the $X$-ray tube $B$ reflects the front view of the square-section shaped charge. The circular cross-section shaped charge has a rotationally symmetric structure, and the photos taken by the two X-ray tubes reflect the same view.

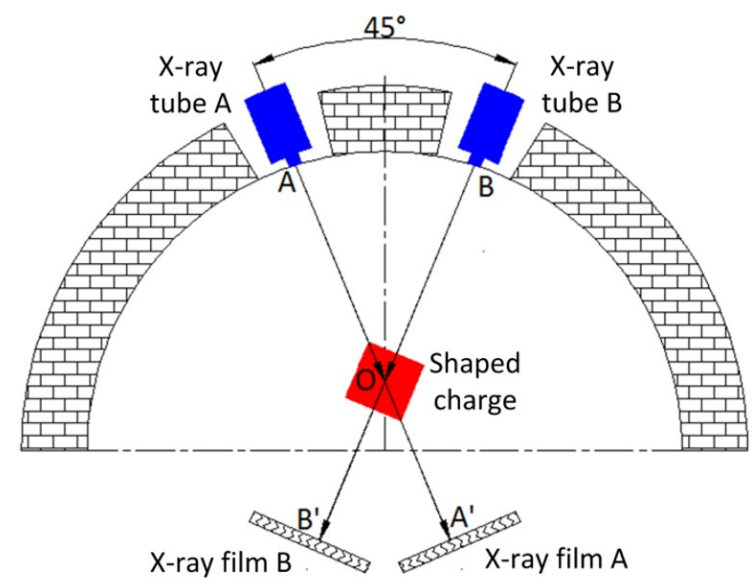

(a) square cross-section shaped charge

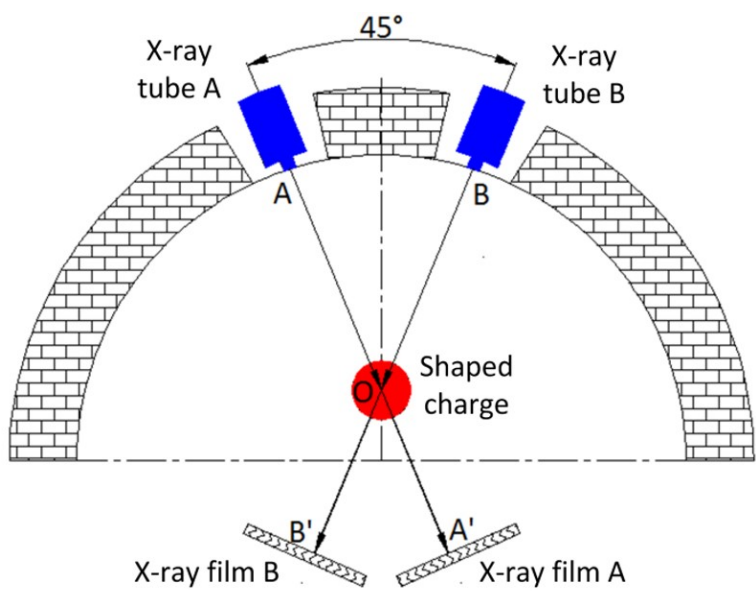

(b) circular cross-section shaped charge

Figure 3 Relative position of the configurations in X-ray experiment.

\subsection{DOP test setup}

The experimental setup of the DOP test is illustrated in Figure 4. It includes a detonator, a booster charge, a base for the booster charge, a main charge, a standoff, and targets. The standoff was made of non-metallic materials. The material of the targets is $45^{\#}$ steel. The DOP tests were conducted at standoffs 80 and $160 \mathrm{~mm}$. 


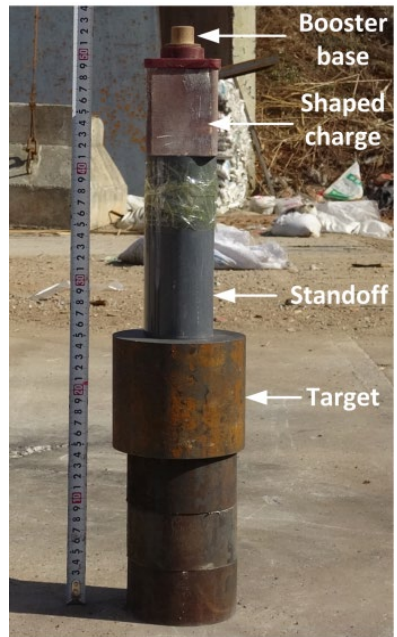

Figure 4 Experimental setup of DOP tests

\section{EXPERIMENTAL RESULTS AND DISCUSSION}

\subsection{Analysis of X-ray photos}

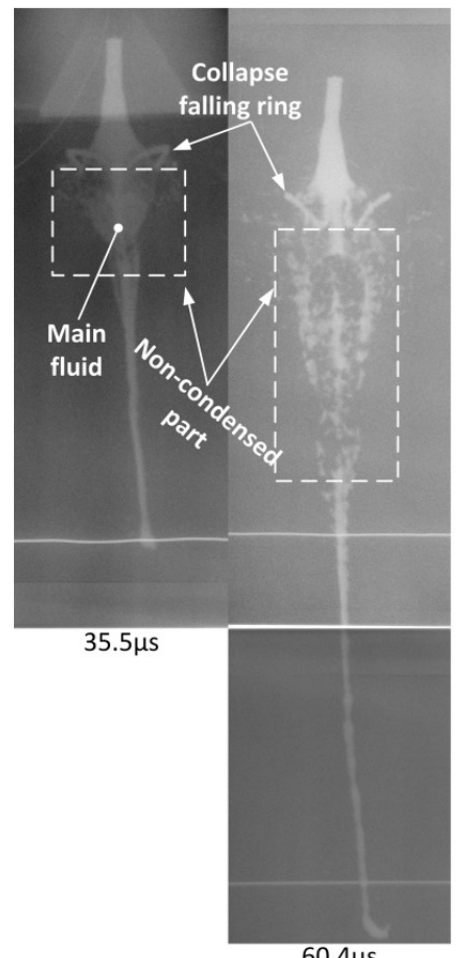

$60.4 \mu \mathrm{s}$

(a) square cross-section shaped charge

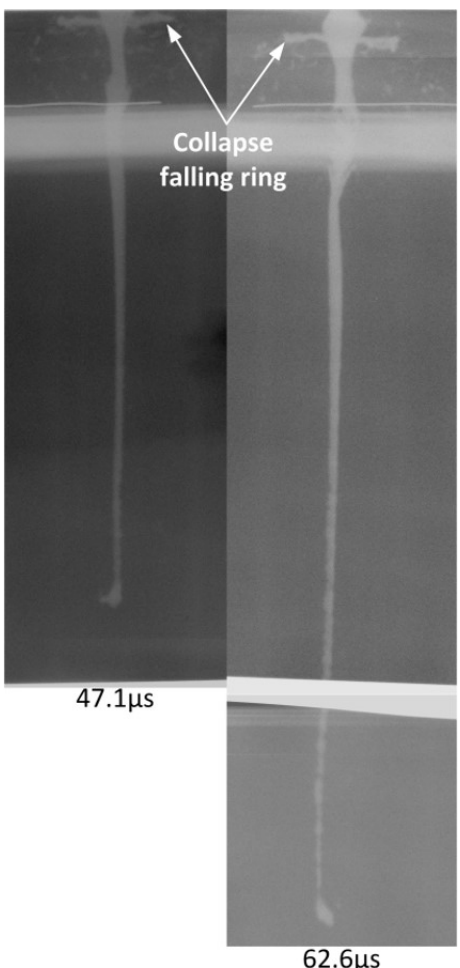

(b) circular cross-section shaped charge

Figure $5 \mathrm{X}$-ray photos of the jets

The diagonal view of the jet morphology of the square cross-section shaped charge at $35.5 \mu$ after detonation and the front view at $60.4 \mu$ s after detonation are shown in Figure 5(a). The jet morphology of the circular cross-section shaped charge at 47.1 and $62.6 \mu$ s after detonation are shown in Figure 5(b). The jet head velocity and jet tail velocity of the square cross-section shaped charge are 5.59 and $1.22 \mathrm{~km} / \mathrm{s}$, respectively, while those of the circular cross-section shaped charge are 5.90 and $1.22 \mathrm{~km} / \mathrm{s}$, respectively.

The X-ray photos demonstrated that the jet formed by the circular cross-section shaped charge is always condensed, and accumulation at the jet head was observed. Neck fracture was also observed in the front segment of the jet at $62.6 \mu \mathrm{s}$. The jet of the square cross-section shaped charge is composed of a condensed part and a non-condensed part, 
and the jet morphology of the condensed part is similar to the corresponding part of the jet of the circular cross-section shaped charge. The non-condensed part is distributed at the jet tail, and the closer to the jet tail, the greater the degree of non-cohesion. The non-condensed part of the jet is composed of a main fluid and off-axis fluids with off-axis velocity in different directions. With the jet stretching, the non-condensed degree of the non-condensed part increases.

The X-ray photos also illustrated that the collapse falling ring formed by the circular cross-section shaped charge is in a circular shape, while the diagonal view of the collapse falling ring formed by the square cross-section shaped charge is in petal-like shape and the front view is linear. This finding indicated that the liner elements of the collapse falling ring of the circular cross-section shaped charge have the same collapse velocity, while the those of the square cross-section shaped charge have different collapse velocities.

\subsection{Analysis of DOP test results}

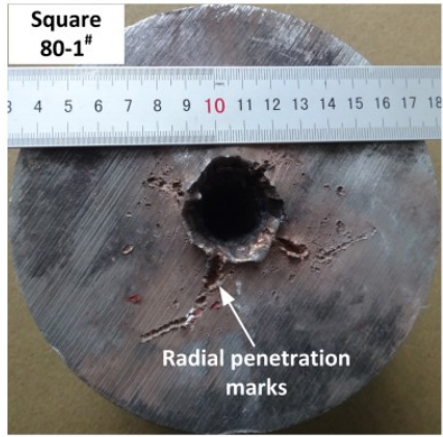

(a) square cross-section shaped charge (standoff $80 \mathrm{~mm}$ )
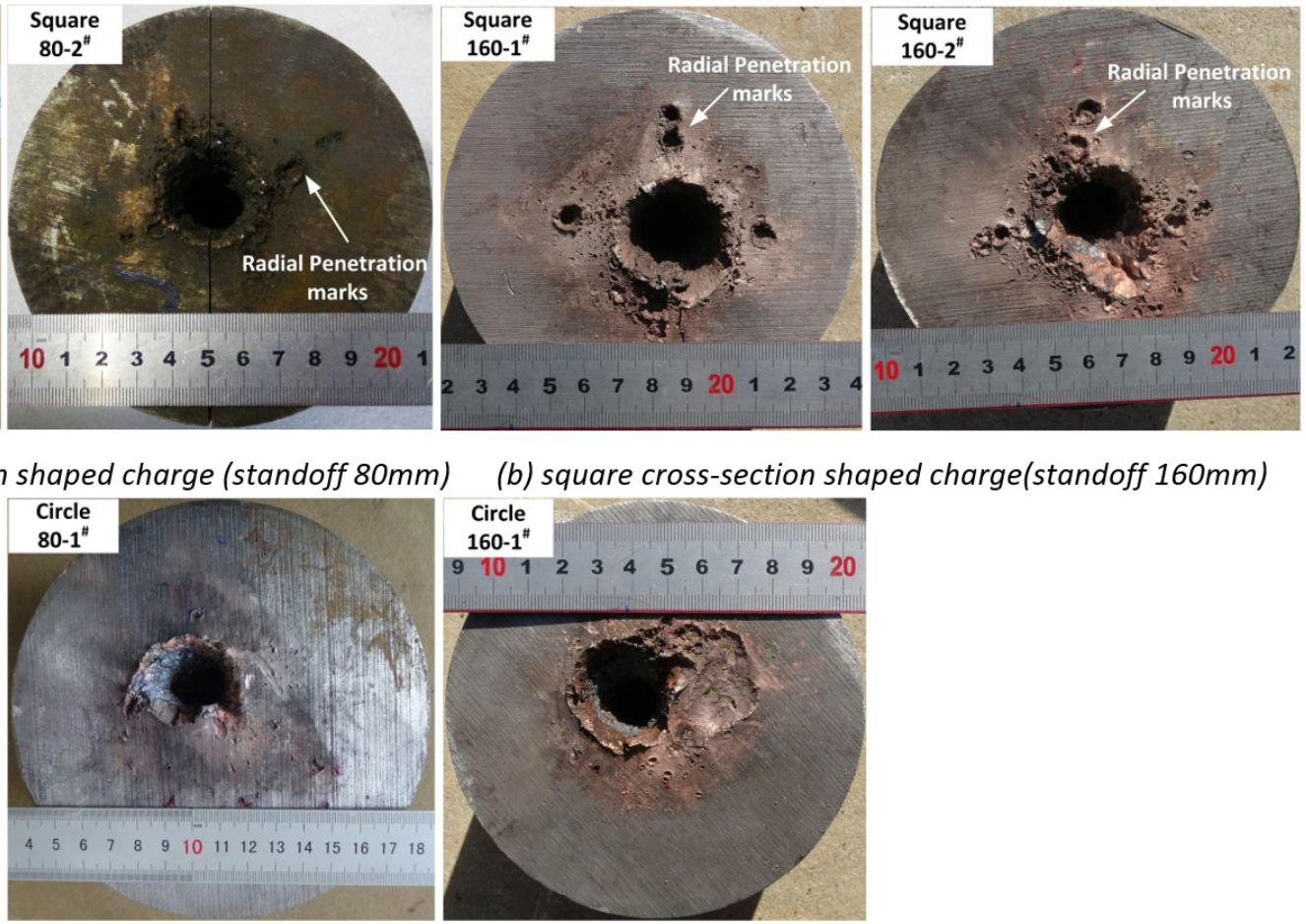

(b) square cross-section shaped charge(standoff 160mm)

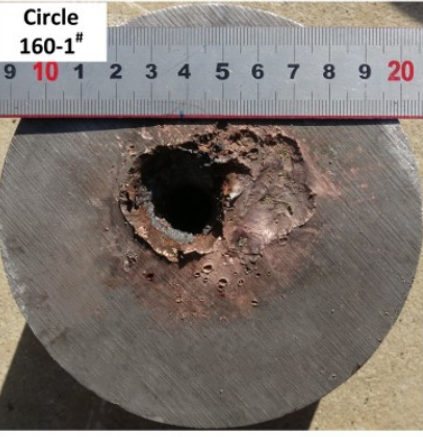

(c) circular cross-section shaped charge

Both shaped charges formed circular entrance holes in the first target, as shown in Figure 6. However, four radial penetration marks were observed around the penetration hole of the square cross-section shaped charge, and the angle between adjacent radial penetration marks was approximately $90^{\circ}$. The penetration marks could be caused by off-axis fluid with high off-axis velocity, and then it can be inferred that there are four off-axis fluids, and the angle between adjacent off-axis fluids is $90^{\circ}$.

Table 1 Entrance hole data

\begin{tabular}{|c|c|c|c|}
\hline Standoff & number & square & circular \\
\hline \multirow[t]{3}{*}{$80 \mathrm{~mm}$} & 1\# & 34 & 30 \\
\hline & $2 \#$ & 32 & - \\
\hline & average & 33 & 30 \\
\hline \multirow[t]{3}{*}{$160 \mathrm{~mm}$} & 1\# & 33 & 30.5 \\
\hline & $2 \#$ & 32 & - \\
\hline & average & 32.5 & 30.5 \\
\hline
\end{tabular}

As listed in Table 1, the average diameter of the entrance hole of square cross-section shaped charge at a standoff of $80 \mathrm{~mm}$ is $33 \mathrm{~mm}$, and that of circular cross-section shaped charge is $30 \mathrm{~mm}$. The average diameter of the entrance 
hole of the square cross-section shaped charge at a standoff of $160 \mathrm{~mm}$ is $32.5 \mathrm{~mm}$, and that of the circular cross-section shaped charge is $30.5 \mathrm{~mm}$. The comparison showed that the entrance hole diameter of the square cross-section shaped charge is slightly larger than that of the circular cross-section shaped charge.

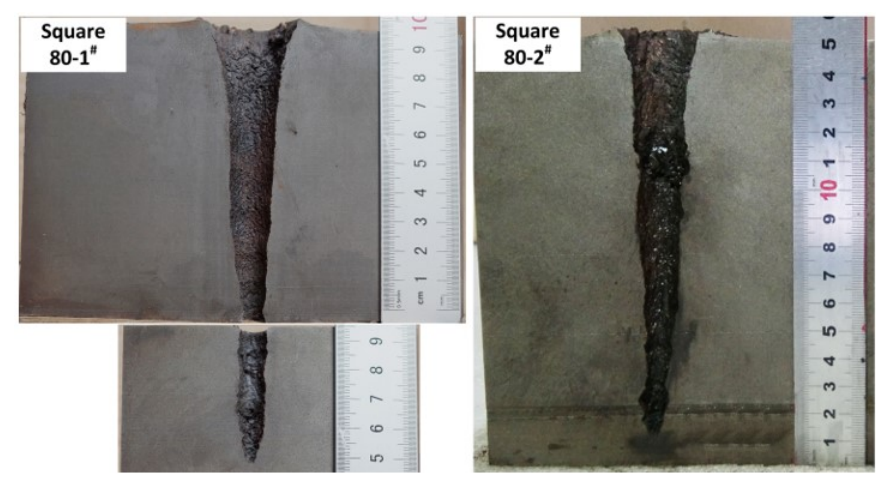

(a) square cross-section shaped charge (standoff $80 \mathrm{~mm}$ )

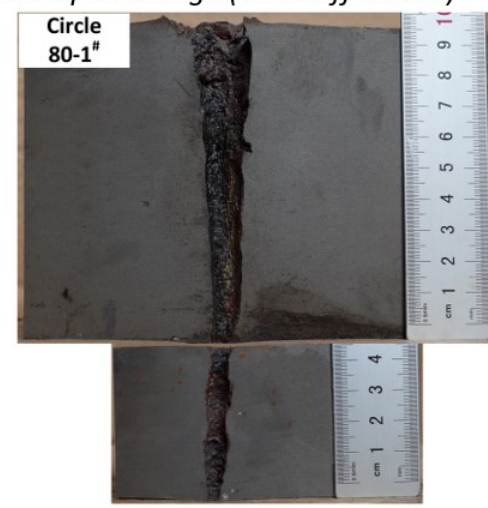

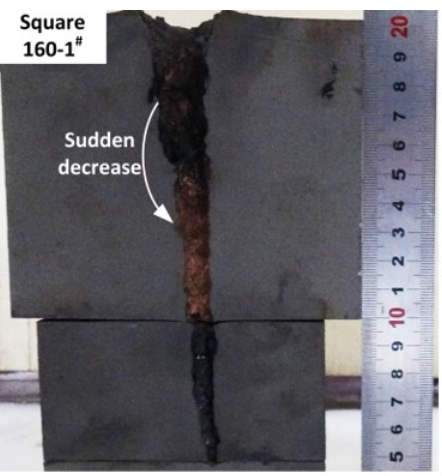

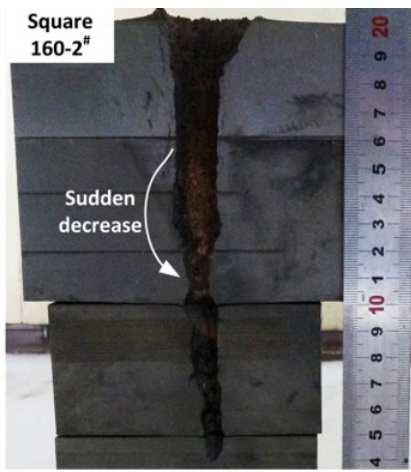

(b) square cross-section shaped charge(standoff $160 \mathrm{~mm}$ )

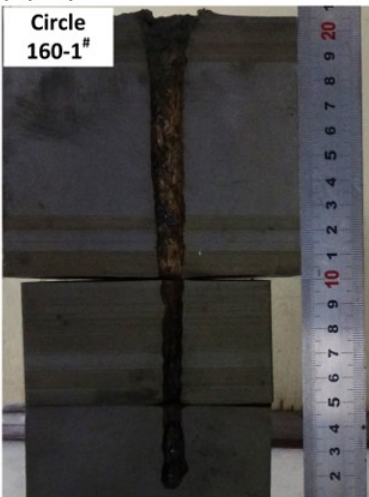

(c) circular cross-section shaped charge

Figure 7 Photographs of the penetration channel

Photographs of the penetration channel of DOP tests are shown in Figure 7. When the standoff is $80 \mathrm{~mm}$, both shaped charges formed a smooth penetration channel, and the jet was slightly fractured in the final penetration stage. When the standoff is $160 \mathrm{~mm}$, the penetration process of the circular cross-section shaped charge remained stable, although the jet is fractured in the final penetration stage. The fractured jet particles did not show any obvious instability phenomenon, such as rotating and off-axis movement. The rough penetration channel formed by the square crosssection shaped charge at a standoff of $160 \mathrm{~mm}$ indicates that instability phenomena, such as rotating and off-axis movement, occurred in the fractured jet particles. In addition, when the standoff is $160 \mathrm{~mm}$, a sudden decrease in the size of the penetration channel of the square cross-section shaped charge was observed, because at this standoff, the off-axis fluid has accumulated a large off-axis distance before reaching the target plate. The off-axis fluid could quickly reach the wall of penetration hole and a second penetration occurs, resulting in an increase in the size of the part of the penetration channel that is secondly penetrated.

Table 2 Penetration depth data

\begin{tabular}{cccc}
\hline Standoff & number & square & circular \\
\hline $80 \mathrm{~mm}$ & $1 \#$ & 147 & 150 \\
& $2 \#$ & 145 & - \\
& average & 146 & 150 \\
& $1 \#$ & 151 & 188 \\
& $2 \#$ & 160 & - \\
& average & 155.5 & 188 \\
\hline
\end{tabular}

As listed in Table 2, the average penetration depth of the square cross-section shaped charge at a standoff of $80 \mathrm{~mm}$ is $146 \mathrm{~mm}$, and that of the circular cross-section shaped charge is $150 \mathrm{~mm}$. The average penetration depth of the square 
cross-section shaped charge at a standoff of $160 \mathrm{~mm}$ is $155.5 \mathrm{~mm}$, and that of the circular cross-section shaped charge is $188 \mathrm{~mm}$. The penetration ability of the square cross-section shaped charge is lower than that of the circular cross-section shaped charge. The decline rate of penetration depth increased from $2.67 \%$ to $17.3 \%$ when the standoff is increased from $80 \mathrm{~mm}$ to $160 \mathrm{~mm}$. The reason for the decrease in penetration ability is that the diameter of the main fluid of the noncondensed part is small. Thus, it could be fractured earlier than the circular cross-section shaped charge at the corresponding position. When the standoff is small, the difference in fracture time has a slight effect on the penetration ability. With the increase in the standoff, instability phenomena, such as rotating and off-axis movement, occur in the fractured jet particles, and the penetration ability of the square-section shaped charge is reduced more obviously.

\section{NUMERICAL SIMULATION}

\subsection{Establishment of simulation model}

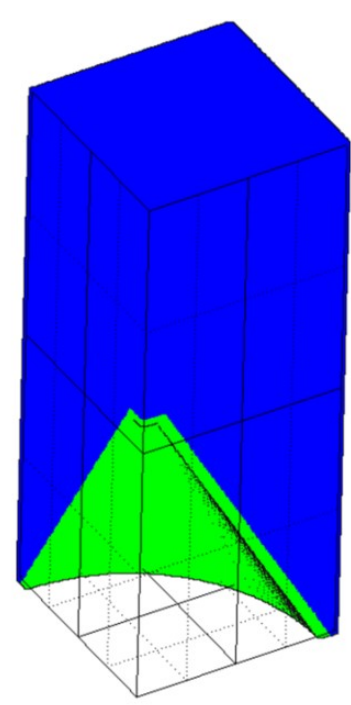

(a) square cross-section shaped charge

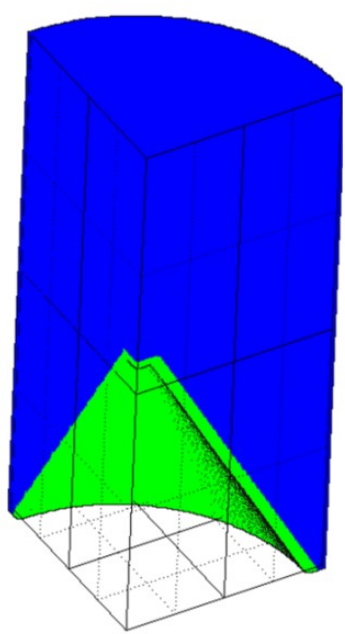

(b) circular cross-section shaped charge

Figure 8 Numerical simulation model

First, three-dimensional simulation models composed of Lagrangian grids was established by using ANSYS/LS-DYNA software. Due to the symmetry, a quarter model was established to improve the calculation efficiency. The finite element model was composed of two parts: explosive and liner. Then, the final solution file was generated and AUTODYN software was used to convert the Lagrangian grids into smoothed particle hydrodynamic (SPH) particles. The smaller the particle size, the more particles were generated and the longer the calculation time. With the decrease of particle size from $1.0 \mathrm{~mm}$ to $0.2 \mathrm{~mm}$, the convergence of the solution was observed. As the particle size is further reduced, the calculation efficiency was greatly reduced. Therefore, the particle size was set to $0.2 \mathrm{~mm}$. The numerical simulation models after the Lagrangian grids were converted to SPH particles are shown in Figure 8.

Composition B was used for explosive; it is described by the MAT_HIGH_EXPLOSIVE_BURN material model and Jones-Wilkins-Lee state equation, and the parameters are shown in Table 3. OFHC Cu was used for liner; it is described by the MAT_JOHNSON_COOK material model and GRUNEISEN state equation, and the parameters are shown in Table 4.

Table 3 The parameters of Composition B [6]

\begin{tabular}{cccccccccc}
\hline parameter & $\rho_{0} / \mathbf{g} \cdot \mathrm{cm}^{-\mathbf{3}}$ & $\boldsymbol{D} / \mathrm{m}^{-\mathbf{s}^{-1}}$ & $\boldsymbol{e}_{0} / \mathrm{KJ} \cdot \mathrm{m}^{-\mathbf{3}}$ & $\boldsymbol{P}_{\mathrm{cJ}} / \mathrm{GPa}$ & $\boldsymbol{A} / \mathrm{GPa}$ & $\boldsymbol{B} / \mathrm{GPa}$ & $\boldsymbol{\omega}$ & $\boldsymbol{R}_{\mathbf{1}}$ & $\boldsymbol{R}_{\mathbf{2}}$ \\
\hline value & 1.63 & 7700 & $8.1 \mathrm{E}+6$ & 25 & 557.48 & 7.83 & 0.34 & 4.5 & 1.2 \\
\hline
\end{tabular}

Table 4 The parameters of OFHC Cu [7]

\begin{tabular}{|c|c|c|c|c|c|c|c|c|}
\hline parameter & $\rho / g \cdot \mathrm{cm}^{-3}$ & $E / G P a$ & G/GPa & $u$ & $A / \mathrm{MPa}$ & $\mathrm{B} / \mathrm{MPa}$ & $n$ & $C$ \\
\hline value & 8.96 & 1.38 & 47.7 & 0.35 & 90 & 290 & 0.31 & 0.02 \\
\hline parameter & $m$ & $T_{M} / \mathrm{K}$ & $T_{R} / \mathrm{K}$ & $\mathrm{C} / \mathrm{km} \cdot \mathrm{s}^{-1}$ & $S_{1}$ & $S_{2}$ & $a$ & $V_{0}$ \\
\hline value & 1.09 & 1360 & 293 & 3.94 & 1.49 & 0 & 0 & 1.99 \\
\hline
\end{tabular}




\subsection{Validation of numerical simulation method}

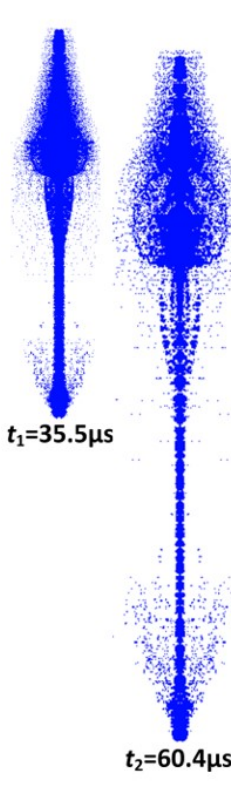

(a) square cross-section shaped charge
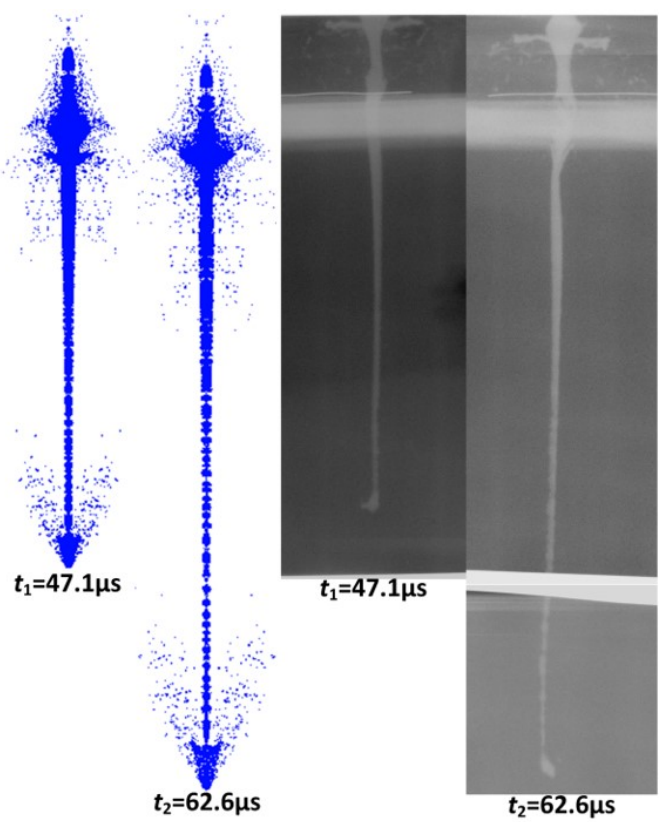

(b) circular cross-section shaped charge

Figure 9 Numerical simulations results (left) and X-ray photos (right)

The numerical results and X-ray photos are shown in Figure 9. The jet morphologies of the numerical simulation and $\mathrm{X}$-ray photos are similar. As listed in Table 5, the numerical results of jet length are close to the experimental ones, the maximum deviation is $10.0 \%$, and the minimum deviation is $-0.8 \%$. The jet head and tail velocities of the square crosssection shaped charge are 6.10 and $1.35 \mathrm{~km} / \mathrm{s}$, respectively, and the deviations from the experiment results are $9.1 \%$ and $10.7 \%$ respectively. The jet head and tail velocities of the circular cross-section shaped charge are 6.15 and $1.25 \mathrm{~km} / \mathrm{s}$ respectively, and the deviations from the experiment results are $4.2 \%$ and $2.5 \%$, respectively. The numerical simulation could reflect the jet formation of the square and circular cross-section shaped charges.

Table 5 Comparison of numerical and experimental results

\begin{tabular}{|c|c|c|c|c|c|c|c|c|}
\hline \multirow{2}{*}{ parameter } & \multicolumn{2}{|c|}{ Jet head velocity $/ \mathrm{km} \cdot \mathrm{s}^{-1}$} & \multicolumn{2}{|c|}{ Jet tail velocity $/ \mathrm{km} \cdot \mathrm{s}^{-1}$} & \multicolumn{2}{|c|}{ Jet length at $t_{1} / \mathrm{mm}$} & \multicolumn{2}{|c|}{ Jet length at $t_{2} / \mathrm{mm}$} \\
\hline & square & circular & square & circular & square & circular & square & circular \\
\hline experiment & 5.59 & 5.90 & 1.22 & 1.22 & 130 & 188 & 239 & 261 \\
\hline simulation & 6.10 & 6.15 & 1.35 & 1.25 & 120 & 192 & 237 & 287 \\
\hline deviation & $9.1 \%$ & $4.2 \%$ & $10.7 \%$ & $2.5 \%$ & $-7.7 \%$ & $2.1 \%$ & $-0.8 \%$ & $10.0 \%$ \\
\hline
\end{tabular}

\subsection{Numerical simulation results and analysis}

\subsubsection{Propagation of detonation and surface rarefaction waves}

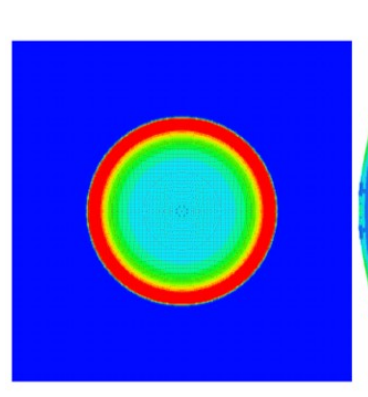

(a) square cross-section shaped charge

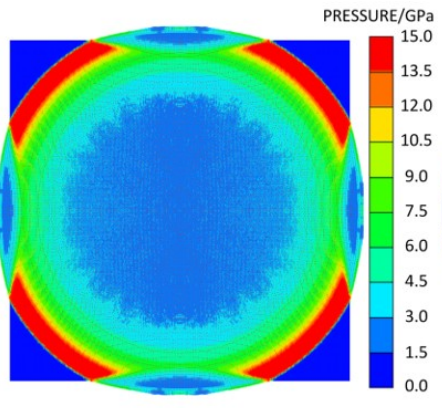

Figure 10 Pressure change on charge cross-section 
The pressure change process on the charge cross-section of the square cross-section and circular shaped charges is shown in Figure 10. The shaped charge was detonated at the center point, and the detonation wave propagated with a spherical shape. After the detonation wave reached the charge surface, a series of surface rarefaction waves began to enter the detonation products, resulting in reduced pressure on the detonation product [8]. The circular cross-section shaped charge had a rotationally symmetric structure, and the surface rarefaction waves entered the detonation products on the same charge cross-section at the same time. Thus, the pressure on the charge cross-section of the circular cross-section is always distributed rotationally symmetrically, as shown in Figure 10(b). The square cross-section shaped charge has a non-rotationally symmetric structure, and differences existed in the time of the surface rarefaction wave entering the detonation products on the same charge cross-section. The detonation wave always reaches the charge surface of the symmetry plane first and the charge surface of the diagonal plane last. Therefore, the pressure of the detonation product near the symmetry plane always decreases faster than the pressure of the detonation product near the diagonal, as shown in Figure 10(a).

\subsubsection{Liner collapse}
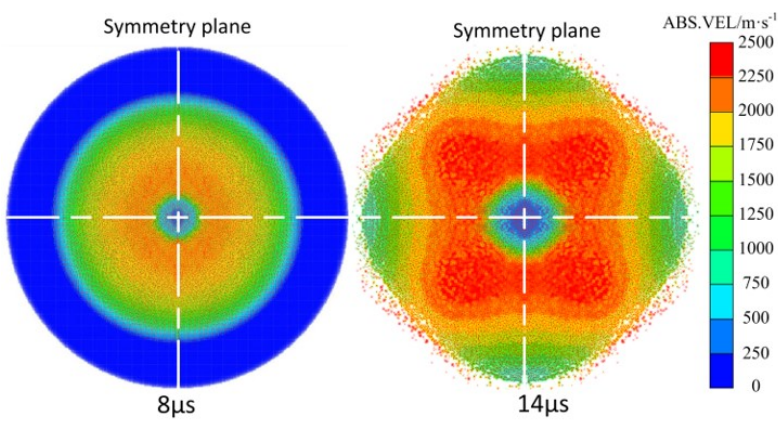

(a) square cross-section shaped charge
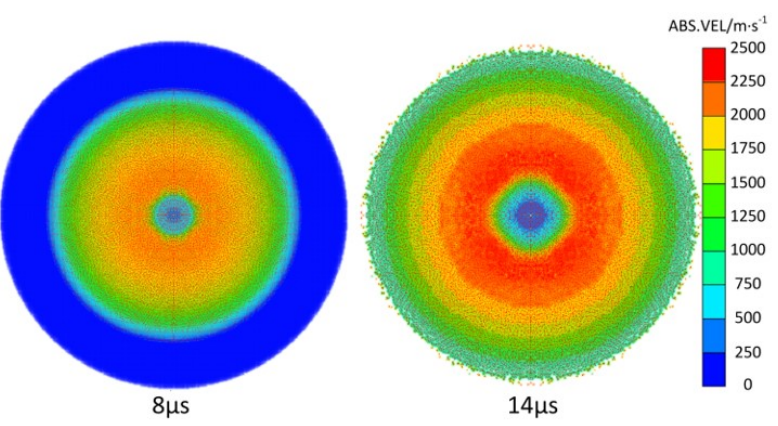

(b) circular cross-section shaped charge

Figure 11 Collapse velocity of liner particles at 8 and $14 \mu \mathrm{s}$

At 8 and $14 \mu$ s, the collapse velocity distribution of the liner particles of the square cross-section and circular crosssection shaped charges is shown in Figure 11. At the initial stage of liner collapse, the surface rarefaction wave could not arrive at the liner surface, and the pressure on the liner surface is distributed in rotational symmetry. Therefore, at $8 \mu \mathrm{s}$, the collapse velocity of liner particles is distributed in rotational symmetry. In the later stage of the liner collapse, the liner collapse is affected by the surface rarefaction wave. For the circular cross-section shaped charge, the time for the surface rarefaction wave enters the detonation product of the same charge cross-section is the same. The surface rarefaction wave arrives at the liner particle surface of the same cross-section at the same time. Therefore, at $14 \mu \mathrm{s}$, the collapse velocity of the particles is still distributed in rotational symmetry. For the square cross-section shaped charge, the surface rarefaction always enters the detonation product near the symmetry plane of the same cross-section first and near the diagonal plane of the same cross-section last. Thus, the pressure of the detonation product on the liner particle surface near the symmetry plane always decreases faster than the pressure of the detonation product on the liner particle surface near the diagonal plane, resulting in the collapse velocity of the liner particle near the diagonal plane higher than that near the symmetry plane at $14 \mu \mathrm{s}$.

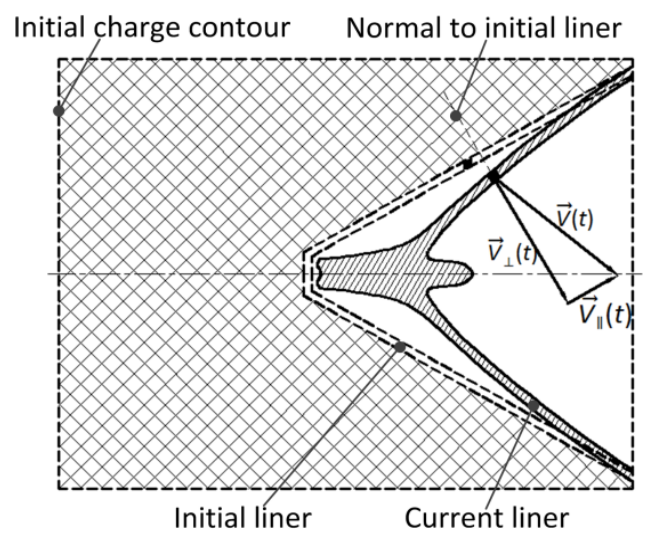

Figure 12 Components of collapse velocity of liner element in the profile 
The collapse of liner element is also affected by the adjacent liner elements [9]. In the axial direction, adjacent liner elements have different collapse velocities, and a force component is generated along the direction of the liner contour [9-10]. As seen from the profile, the collapse velocity has a component $\left(\vec{V}_{\|}(t)\right)$ parallel to the liner contour (Figure 12).

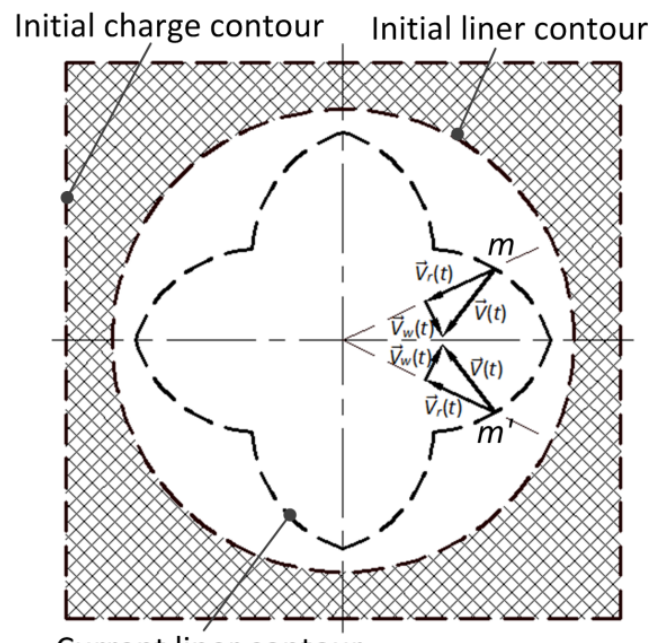

Current liner contour

Figure 13 Components of collapse velocity of liner element in cross section

For the square cross-section shaped charge, the adjacent liner elements also experience different collapses in the circumferential direction, and a force component in the tangential direction of the liner is generated [11]. As seen from the cross-section, the collapse velocity has a component $\left(\vec{V}_{w}(t)\right)$ perpendicular to the diameter direction (Figure 13).

\subsubsection{Jet formation}

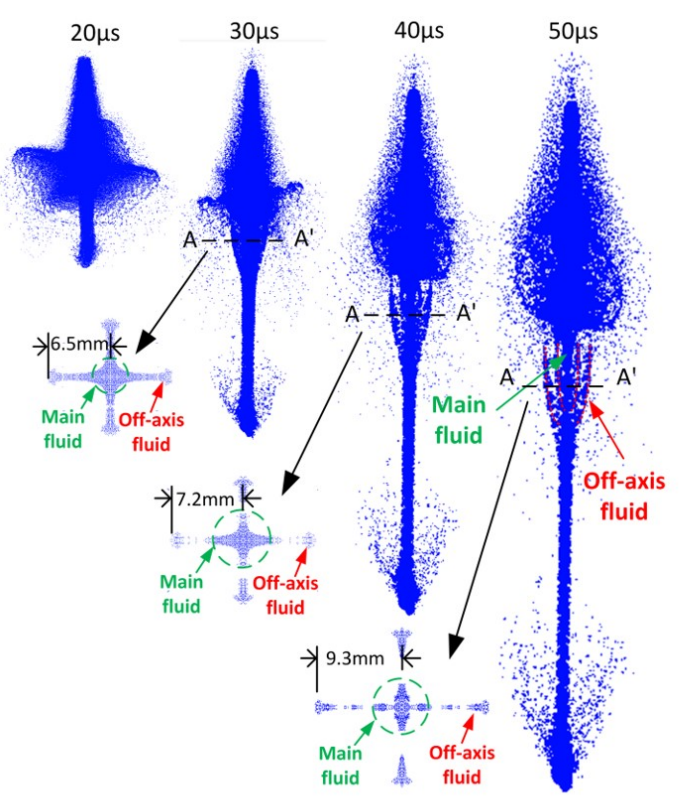

(a) square cross-section shaped charge (oblique side view)
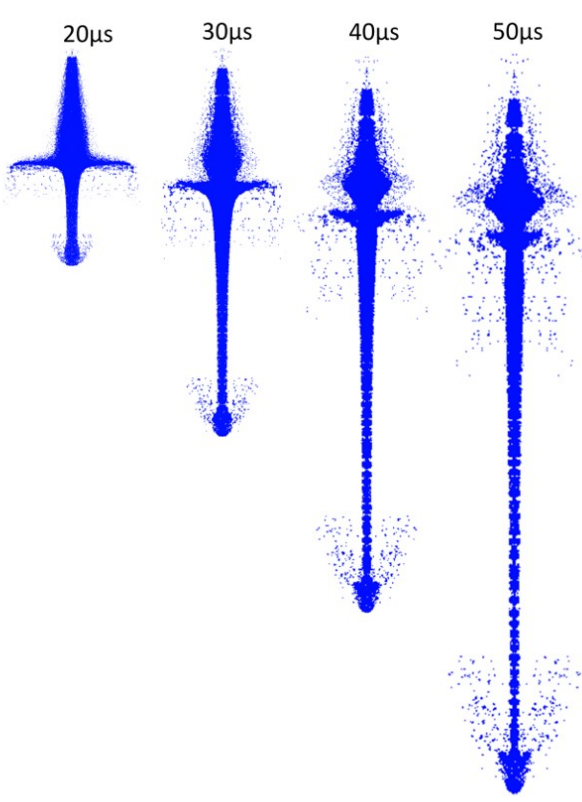

(b) circular cross-section shaped charge

Figure 14 Jet formation of shaped charges

The jet formation of the square and circular cross-section shaped charges is shown in Figure 14. The jet formed by the circular cross-section shaped charge is always condensed, whereas that formed by the square cross-section shaped charge experienced a non-condensed phenomenon near the jet tail, consistent with the X-ray experiment results. Fig.14 (a) shows the shape and the shape change process of the $A A^{\prime}$ cross-section of the non-condensed part. This part of the jet is composed of a main fluid and four off-axis fluids, where the main fluid is distributed along the nominal axis and moving along the nominal axis. The four off-axis fluids are distributed at a certain distance from the nominal axis, and 
they are on the symmetry plane. From $30 \mu$ s to $50 \mu \mathrm{s}$, the maximum off-axis distance of the off-axis fluid on the AA' crosssection increased from $6.5 \mathrm{~mm}$ to $7.2 \mathrm{~mm}$ and finally to $9.3 \mathrm{~mm}$, indicating that the off-axis fluid has an off-axis velocity along the symmetry plane. The closer the position to the jet tail, the more serious the degree of jet non-cohesion, indicating that the off-axis velocity of the off-axis fluid is greater.

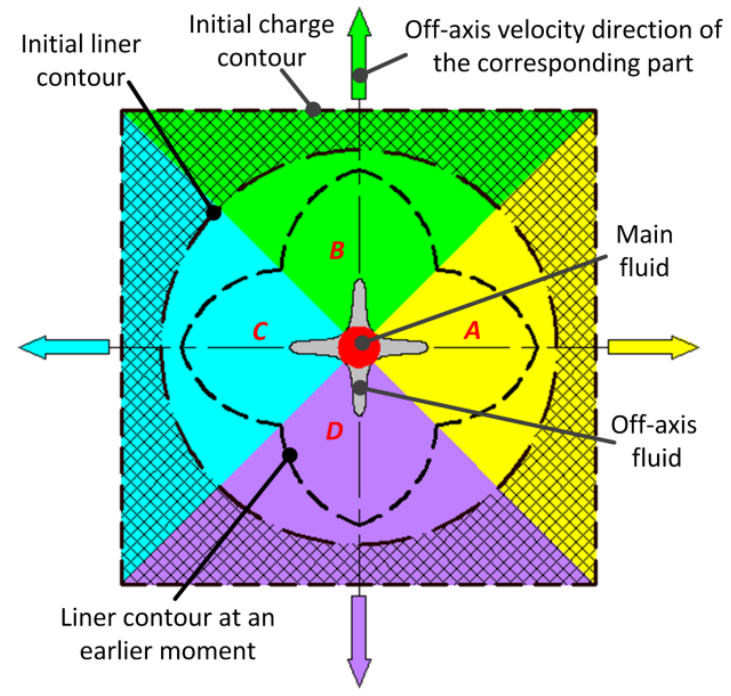

Figure 15 Schematic of the formation of jet element of the non-condensed part

The front segment of the jet is formed by liner elements near the liner top [10]. These liner elements are close to the nominal axis, and the difference in the time for the surface rarefaction wave to enter the detonation product has a slight effect on the collapse of these liner elements. Therefore, the jet morphology of the front segment of the jet is similar to the corresponding part of the jet of the circular cross-section shaped charge.

The jet tail is formed by liner elements near the liner bottom [12]. The time difference for the surface rarefaction wave to enter the detonation product causes the liner elements on the same cross-section of these position to experience remarkable different collapses. Figure 15 illustrates the formation of jet element when a difference exists in the liner element collapse of the same cross-section. When the liner element near the diagonal plane with a higher collapse velocity arrived in the nominal axis and formed a main jet, the liner element near the symmetry plane with a lower velocity did not arrive in the nominal axis and finally formed the off-axis fluid. The off-axis fluid is distributed on the symmetry plane. The collapse velocity component $\vec{v}_{w}(t)$ makes the off-axis fluid have an off-axis velocity along the symmetry plane. Therefore, as time goes by, the degree of non-condensation of the jet increases. As shown in Figure 15, the square cross-section shaped charge is divided into four parts: A, B, C, and D. Each part has the same structural characteristics. Each part generates one off-axis fluid, and the velocity direction of the off-axis fluid is shown in Figure 15. The closer to the liner bottom, the greater the difference in the liner element collapse of the same cross-section, and the greater the velocity component $\vec{V}_{\mathrm{w}}(\mathrm{t})$. Therefore, the closer to the jet tail, the higher off-axis velocity of the off-axis fluid, and the more serious the non-condensation phenomenon is.

\subsubsection{Influence of inscribed circle size on jet formation}

Previous analysis showed that for the square-section shaped charge, the time for the surface rarefaction wave to enter the detonation product of the same cross-section is different, and the liner elements near the liner bottom are greatly affected, which leads to non-condensation phenomenon at the jet tail. By keeping the liner structure and increasing the inscribed circle diameter of the charge cross-section, the time for the surface rarefaction wave to enter the detonation product could be delayed and the influence on the liner elements near the liner bottom could be weakened, thereby avoiding the non-condensation phenomenon of the jet.

The jet formation of the square cross-section shaped charges with different inscribed circle diameters is shown in Figure 16. When the inscribed circle diameter of the charge section increases from $56 \mathrm{~mm}$ to $61.6 \mathrm{~mm}$ (1.1 times of $56 \mathrm{~mm}$ ), the degree of non-cohesion at the jet tail decreases. When the inscribed circle diameter of the charge section is greater than $67.2 \mathrm{~mm}$ (1.2 times of $56 \mathrm{~mm}$ ), the square-section shaped charge forms a condensed jet. 


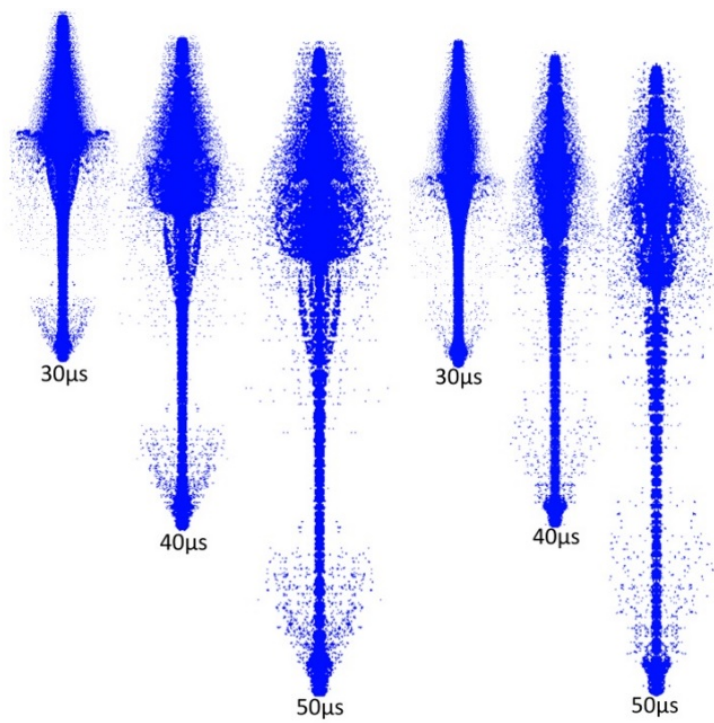

(a) the inscribed circle diameter is $56 \mathrm{~mm}$

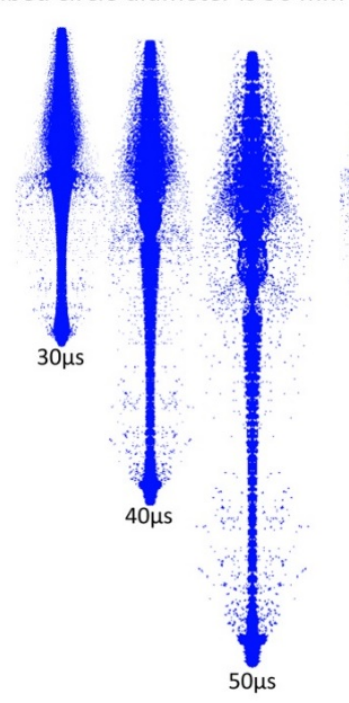

(c) the inscribed circle diameter is $67.2 \mathrm{~mm}$

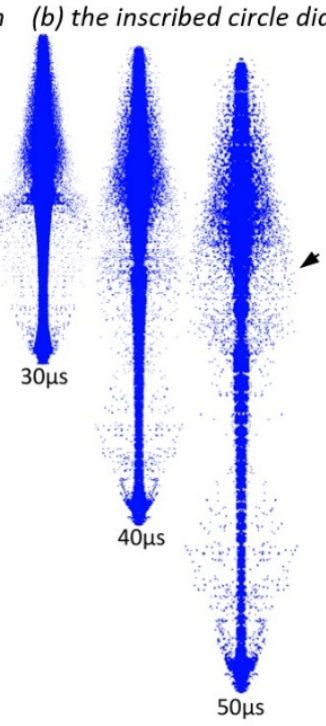

(d) the inscribed circle diameter is $72.8 \mathrm{~mm}$

Figure 16 Influence of inscribed circle size on jet formation of square cross-section shaped charge

\section{CONCLUSION}

In this work, X-ray experiments were carried out for the jet formation of square cross-section shaped charge with inscribed circle diameters of $56 \mathrm{~mm}$. DOP tests at standoffs 80 and $160 \mathrm{~mm}$ were also conducted. The experimental results are compared with the circular cross-section shaped charge with same inscribed circle diameter. Related numerical simulations were carried out on the basis of SPH method. The conclusions drawn are as follows.

(1) The jet formed by the circular cross-section shaped charge is always condensed, whereas the jet of the square crosssection shaped charge is composed of a condensed part and a non-condensed part. The jet morphology of the condensed part is similar to the corresponding part of the jet of the circular cross-section shaped charge. The noncondensed part is distributed at the jet tail, and it is composed of a main fluid and four off-axis fluids with off-axis velocity along the symmetry plane. The closer the position is to the jet tail, the greater the off-axis velocity of the off-axis fluid, and the more serious the degree of jet non-cohesion.

(2) The size of the entrance hole of the square cross-section shaped charge is slightly larger than that of the circular cross-section shaped charge at the same standoff. When the standoff is $160 \mathrm{~mm}$, a sudden decrease in the size of the penetration channel of the square cross-section shaped charge could be observed. 
(3) The non-condensing of the jet makes the penetration ability of the square cross-section shaped charge lower than that of the circular cross-section shaped charge. The decreasing rate of penetration depth increased from $2.67 \%$ to $17.3 \%$ when the standoff is increased from $80 \mathrm{~mm}$ to $160 \mathrm{~mm}$.

(4) By keeping the liner structure and increasing the inscribed circle diameter of the charge cross-section, the noncondensation phenomenon could be avoided by the jet. For the liner structure in this work, when the inscribed circle diameter of the charge cross-section is larger than $67.2 \mathrm{~mm}(1.2$ times of $56 \mathrm{~mm})$, the square cross-section shaped charge forms a condensed jet.

\section{Acknowledgement}

This paper is supported by the National Natural Science Foundation of China (Grant Nos.11972196, 11872214 and 11702144), and Youth fund of Jiangsu Natural Science Foundation (Grant Nos.BK20190433).

Author's Contribuitions: Writing - original draft, Yuting WANG; Test technical support, Zhengxiang HUANG, Xudong Zu; Writing - review \& editing, Bin MA; Numerical simulation technical support, CAI You-er

Editor: Marcílio Alves

\section{References}

[1] Hemsch M J, HONG Jin-sen, YANG Qi-de. Tactical missile aerodynamics [M]. Beijing: Astronautics Press, 1999:367-392.

[2] Pantelatos D K, Tzotzolakis D C, Mathioulakis D S. Two noncircular cross-section bodies and a high wing-body configuration at incidence in a low subsonic free stream[J]. Journal of Fluids and Structures, 2008, 24(6): 778-798.

[3] Ahmed M, Malik A Q. A review of works on shaped charges[J]. Engineering, Technology \& Applied Science Research, 2017, 7(5): 2098-2103.

[4] Cullis I G, Nash M A. Theoretical design of non-symmetrical shaped charge [C]//9th International Symposium on Ballistics.1986: 273-283.

[5] WANG Y T, HUANG ZX, MA B, et al. Study on Jet Formation and Penetration of Square Cross-Section Shaped Charge[C]//31st International Symposium on Ballistics. 2019, 2215-2224

[6] Murphy M J, Lee E L, Weston A M, et al. Modeling shock initiation in composition B[R]. Lawrence Livermore National Lab, CA (United States), 1993.

[7] Li W, Wang X, Li W. The effect of annular multi-point initiation on the formation and penetration of an explosively formed penetrator[J]. International Journal of Impact Engineering, 2010, 37(4): 414-424.

[8] SUN Cheng-wei, WEl Yu-zhang, ZHOU Zhi-kui. Applied detonation physics[M]. Beijing: National Defense Industry Press, 2000: 216-239. (In chinese)

[9] Chou P C, Hirsch E, Ciccarelli R D. An Unsteady Taylor Angle Formula for Liner Collapse[R]. DYNA EAST CORP PHILADELPHIA PA, 1981.

[10] Chou P C, Carleone J, Flis W J, et al. Improved formulas for velocity, acceleration, and projection angle of explosively driven liners[J]. Propellants, explosives, pyrotechnics, 1983, 8(6): 175-183.

[11] WANG Yu-ting, HUANG Zheng-xiang, JIA Xin, et al. Jet Formation and Penetration Characteristics of Shaped Charge with Elliptical Cross-section [J]. Chinese Journal of Energetic Materials(Hanneng Cailiao), 2021, 29(2):96-106. (In chinese)

[12] HUANG Zheng-xiang, ZU Xu-dong. Terminal Effects [M]. Science Press, 2014:136-153. (In chinese) 